\title{
Référentiels de durabilité forestière : l'universalité en question
}

\author{
Hervé Brédif \\ Agronome et géographe, CNRS UMR 7533, Institut de géographie, 75005 Paris, France
}

\begin{abstract}
H. Brédif revient sur un thème déjà abordé dans l'ouvrage Quelles forêts pour demain? qu'il a cosigné avec Pierre Boudinot (cf. NSS, $\left.2002,10, n^{\circ} 2\right)$. Il analyse ici les logiques qui président à la mise en place de dispositifs supposés traduire concrètement les principes du développement durable. Il montre notamment que l'universalité du système pose question.

Cet article et celui de A. Karsenty et R. Pirard (NSS, 2007, 15, $\mathrm{n}^{\circ} 4$ ) offrent au lecteur un panorama de deux des principaux débats qui ont traversé les réunions internationales sur la forêt au cours des dernières années et des dérives possibles entre intention et mise en œuvre.
\end{abstract}

La Rédaction

\begin{abstract}
Mots-clés :
forêts ;

durabilité ;

processus processus les États, l'autre par des acteurs privés, structurent la pensée et l'action en matière de durabilité forestière.
intergouvernementaux; La logique fondamentale suivie par ces dernières s'avère pourtant étonnamment proche : en cherchant à ONG et systèmes de certification ; référentiels de durabilité

Résumé - Le recours immodéré à la notion de la durabilité masque souvent une réflexion insuffisante quant aux conditions nécessaires à la mise en œuvre des processus qui s'y rattachent. La question forestière s'avère à cet égard particulièrement instructive. Deux grandes démarches internationales, l'une portée par donner un contenu précis à la durabilité forestière, elles privilégient la qualité intrinsèque des forêts au détriment des besoins des acteurs. En ce sens, elles ne constituent certainement pas une traduction fidèle de l'objectif initial du développement durable. Quant à leur prétention à l'universalité, elle relève en définitive de la pure mystification.
\end{abstract}

\section{Keywords:}

forests; sustainability; intergovernmental processes;

NGOs

and certification systems; sustainability reference bases

\begin{abstract}
Reference Bases for Forest Sustainability: Universality Challenged. The immoderate use of the notion of sustainability often obscures the essential point of the conditions really needed to bring into play the processes that attach to it. Hence a great disappointment due to the gap between the intentions declared and the effects achieved. The forest issue turns out to be highly instructive on that point. As far as the implementation of forest sustainability is concerned, two main international approaches may be observed. Using the logic of principles, criteria and indicators, States aim to define sustainability as precisely as possible. A kind of ideal model of well-managed forest is produced. Consequently, the debate on objectives is replaced with purely technical standards. The other international approach, supported by private actors, is market-driven. Major environmental NGOs have placed great hope in the emergence of this tool supposed to favour a new forest governance. However, its logic is quite similar to the previous one and leads to promote an environmental standard far remote from the initial objective of sustainable development and unable to solve the real problems that forest actors are facing. Finally, despite their claim to offering universal solutions to the problem of sustainability, these two approaches appear in fact to be pure mystifications.
\end{abstract}

\section{Introduction. Le modèle de l'île de Pâques}

"Il y a bien longtemps, l'île de Pâques était couverte de forêts, de villages et de champs. C'était un paradis. Ses habitants construisaient de grandes statues pour honorer leurs ancêtres. Ils les sculptaient dans la pierre et les transportaient sur des rondins de bois. On raconte qu'ils coupèrent tant d'arbres que leur petite île

Auteur correspondant : hbredif@univ-paris1.fr n'eut plus de forêts. Leur civilisation n'est plus, mais les statues sont toujours là. Elles nous rappellent que les énergies qui font les civilisations peuvent aussi les défaire. En consacrant 1 million d'euros par jour à la recherche, EDF développe les énergies de demain. $»^{1}$

\footnotetext{
${ }^{1}$ Le film de la campagne EDF île de Pâques peut être visualisé sur le site : http://www.edf.fr/html/paques/Internet/index.html
} 
Ce petit texte, qui ne l'a lu, qui ne l'a entendu? Qui n'a pas été saisi par sa sobriété, son efficacité? Pendant près d'une année, l'espace publicitaire français a résonné des lointains mystères de l'île de Pâques.

Il ne fait guère de doute que le livre de Jared Diamond (2005) a inspiré les publicitaires d'EDF. Dans son ouvrage, Diamond explique en effet que, dans sa recherche d'identification des facteurs pouvant rendre compte de la disparition ou du maintien des sociétés, l'île de Pâques constitue une allégorie sans pareille de la société-monde contemporaine. Si le modèle de l'île de Pâques fonctionne aussi bien, c'est que le drame s'y joue au travers d'un nombre épuré de facteurs. Sur les cinq grands facteurs explicatifs de l'effondrement des sociétés identifiés par Diamond -1) l'impact qu'exercent par inadvertance les populations sur leur environnement ; 2) le changement climatique ;3) la présence de voisins hostiles ; 4) le soutien de voisins amicaux ; 5) la capacité des sociétés à faire face à leurs problèmes, en y apportant des réponses adaptées -, les trois facteurs du centre peuvent être écartés dans le cas de l'île de Pâques, en raison, d'une part, de son isolement complet au beau milieu du Pacifique et, d'autre part, de l'absence de toute occurrence confirmée de changement climatique. En somme n'auraient joué que le premier facteur, matérialisé par la déforestation à outrance de l'île, et le dernier, à savoir l'incapacité des différents clans résidant sur l'île d'empêcher l'issue mortifère dans laquelle devait les pousser ladite déforestation.

C'est dire le poids symbolique des forêts et la place qu'elles occupent dans l'imaginaire occidental. Si la forêt tropicale humide constitue le symbole par excellence de la biodiversité, la déforestation incarne, mieux que tout autre phénomène, l'idée d'une société humaine assise sur des bases non durables, dont l'espérance de vie s'avère dès lors comptée. Là où disparaît la forêt, la société ne saurait survivre longtemps.

Si les forêts revêtent un caractère emblématique dans le débat sur le développement durable, elles fournissent également un terrain de prédilection pour examiner la manière dont on tente de réaliser la durabilité. En effet, plus que d'autres domaines, la question forestière, depuis le premier Sommet de la Terre (1992), a été envisagée au travers de processus internationaux; depuis, ces derniers n'ont cessé de structurer les réflexions, les initiatives et les recherches au nom de la durabilité forestière; les considérer attentivement, dans leurs caractéristiques et leur logique constitutive en dit long sur la manière dont est envisagé le développement durable, telle est l'hypothèse qui fonde le présent article ${ }^{2}$.

Deux grands ensembles de processus internationaux sont classiquement distingués dans le cas des forêts. Les

\footnotetext{
${ }^{2}$ Les considérations qui suivent s'appuient sur plusieurs années de recherches, conduites en particulier à l'AFOCEL (association Forêt cellulose, organisme de recherche sur la forêt et les produits à base de bois).
}

premiers correspondent à des processus intergouvernementaux ; les seconds renvoient à des démarches privées de certification et de labellisation de produits à base de bois, portées par des associations de défense de l'environnement ou bien des professionnels de la forêt et du bois. Classiquement, les recherches s'orientent selon deux directions principales. Le plus souvent, elles s'interrogent sur les vertus et les faiblesses comparées des différents systèmes de certification forestière, jusqu'à se demander parfois si la certification forestière constitue une réponse adéquate aux grands problèmes forestiers du monde. Les chercheurs en sciences politiques quant à eux retiennent avant tout $\mathrm{l}^{\prime}$ opposition entre processus intergouvernementaux et dispositifs de certification «tirés par le marché » : dans cette optique, la certification forestière est généralement appréhendée comme le symptôme autant que la réponse incomplète à la crise de gouvernance globale qui affecte les forêts du monde (Humphreys, 2006; Guéneau, 2007).

Notre contribution approfondit une autre voie. Elle s'intéresse aux fondements mêmes des processus internationaux évoqués ci-dessus, à savoir la possibilité de définir de manière universelle ce qu'est le développement durable appliqué aux forêts. Nous pensons établir que ces processus procèdent en réalité d'une même logique et s'avèrent tout aussi injustifiés à revendiquer une quelconque valeur universelle.

La logique suivie par les processus intergouvernementaux sera analysée dans une première partie. Nous la retrouverons à l'œuvre, poussée à l'extrême, dans les systèmes de certification forestière; plus particulièrement étudié dans une seconde partie, le cas du Forest Stewardship Council montrera combien la logique de ces faux universels éloigne du questionnement initial du développement durable.

\section{Processus intergouvernementaux : la logique du contenu fiable et universel}

Du milieu des années 1980 au début des années 1990, la déforestation qui sévit en Amazonie suscite une grande émotion, notamment dans les pays anglo-saxons. Aussi, la question forestière hante-t-elle le premier Sommet de la Terre. D'aucuns placent leurs espoirs dans une convention mondiale, ayant force juridique, sur les forêts. Refusant cependant de se voir dicter les conditions de leur développement par les pays riches, plusieurs grands pays forestiers du Sud ${ }^{3}$ récusent cette voie de manière catégorique. Le principe de souveraineté des États en matière de ressources naturelles se trouve même réaffirmé avec force. De sorte que Rio aboutira, dans le domaine forestier,

\footnotetext{
3 À commencer par le Brésil, très vigilant à l'égard de tout ce qui pourrait entraver son modèle de développement agricole et agroalimentaire qui mise avant tout sur l'exportation.
} 
à une simple «déclaration de principes, non juridiquement contraignante, mais faisant autorité pour un consensus mondial sur la gestion, la conservation et le développement durable de tous les types de forêts ». Longue d'une dizaine de pages, cette déclaration s'apparente à une charte générale, dont le titre, à lui seul, en dit long sur l'âpreté des négociations ayant présidé à son établissement. Les différents pays signataires reconnaissent partager un certain nombre de principes communs pour une gestion durable, chacun se réservant le droit de les mettre en application.

Il est néanmoins décidé de poursuivre la réflexion sur les forêts dans une enceinte officielle de haut niveau : un Groupe intergouvernemental sur les forêts (IPF) ${ }^{4}$ réunira une ou deux fois par an les experts dépêchés par les gouvernements. Ces experts expriment rapidement le besoin de donner un contenu plus précis à la durabilité forestière ; les formulations retenues à ce sujet dans la déclaration de principes leur paraissent encore trop sujettes à interprétation. Ainsi s'impose l'idée de recourir à des critères et à des indicateurs.

L'immensité des surfaces concernées (les forêts couvrent un quart des surfaces émergées, soit presque quatre milliards d'hectares) et la variété des conditions biogéographiques conduisent les experts gouvernementaux à proposer une régionalisation du travail. Pas moins de sept processus intergouvernementaux, chacun correspondant à une grande aire géographique et forestière, vont être chargés de prendre le relais (Fig. 1). L'Europe géographique, quant à elle, va s'appuyer sur un organe multipartite dont elle dispose depuis plusieurs années - la conférence ministérielle pour la protection des forêts en Europe - afin d'avancer dans cet objectif de définition de la durabilité forestière à partir de critères et d'indicateurs. Le dynamisme de certains de ses membres - à commencer par les délégations française et finlandaise va conduire ce processus centré sur l'Europe à prendre de facto une sorte de leadership dans la conception des jeux de critères et d'indicateurs. Le processus paneuropéen, comme on l'appellera bientôt, va largement inspirer l'architecture et le contenu des six autres processus régionaux. Dès 1993, il propose sa propre définition de la gestion durable des forêts. L'année suivante, un jeu de six critères et de vingt-sept indicateurs quantitatifs, complété d'indicateurs qualitatifs, est adopté pour les forêts européennes. Le désir de renseigner ces indicateurs en s'appuyant sur des mesures objectives est revendiqué très tôt $t^{5}$. Les documents officiels présentent les critères et

\footnotetext{
${ }^{4}$ L'International Panel on Forests - homologue du Groupe intergouvernemental d'étude du climat (GIEC/IPCC) - fonctionnera de 1995 à 1997, avant d'être remplacé par le Forum intergouvernemental sur les forêts (FIF) de 1997 à 2000, lui-même évoluant en Forum des Nations unies sur les forêts (FNUF).

${ }^{5}$ Le rapport du séminaire intergouvernemental sur les critères et indicateurs tenu à Helsinki en août 1996 retient la définition suivante : «Un indicateur signifie une mesure quantitative,
}

les indicateurs comme un moyen de définir précisément la gestion durable des forêts. Ils doivent servir de matrice générale pour la constitution de jeux de critères et indicateurs nationaux, mais aussi permettre un suivi de l'évolution des situations des forêts et des comparaisons entre pays.

En 1997, lors d'une session de travail de ce processus, certains experts font valoir qu'en toute bonne logique, il conviendrait de décliner ces critères et indicateurs généraux à des échelles de gestion plus fine encore. Après avoir évoqué la parcelle ou la propriété forestière, les délégués des administrations forestières retiennent la notion d'unité de gestion forestière. Réunis à Lisbonne en 1998 pour la troisième conférence ministérielle pour la protection des forêts en Europe, les ministres des différents pays membres adoptent un document intitulé Recommandations paneuropéennes pour une gestion forestière durable au niveau opérationnel. Classées selon les six grands critères du processus paneuropéen, ces recommandations sont regroupées en deux catégories : des recommandations pour la planification de la gestion forestière ; des recommandations plus concrètes, relatives aux pratiques de gestion forestière. Dans les années qui suivent, les experts gouvernementaux éprouvent le besoin d'améliorer les indicateurs : après plusieurs séminaires, ils proposent une nouvelle série de 35 indicateurs quantitatifs, toujours accompagnés d'une ébauche d'indicateurs qualitatifs. Une nouvelle conférence ministérielle réunie à Vienne en 2003 adopte ce jeu révisé d'indicateurs, en même temps qu'elle recommande l'établissement de programmes nationaux forestiers ${ }^{6}$. Les différents pays sont invités à suivre le cadre des critères et indicateurs pour établir ces programmes, présentés comme des documents de planification générale au sujet des forêts. Plusieurs principes méthodologiques généraux sont préconisés (participation, approche holistique et intersectorielle, processus itératif assorti d'un engagement à long terme...); il est également recommandé de suivre une «approche par écosystèmes ».

Â première vue, la démarche suivie s'avère limpide et nul n'oserait en contester la rigueur. N'était-il pas impérieux, en effet, de donner un contenu tangible et certain à la durabilité forestière? Au demeurant, le fait qu'à quelques variantes près, une même approche globale soit adoptée par la plupart des pays et encouragée par les organes internationaux (FIF, FAO, CIFOR, OIBT...) s'avère rassurant. Enfin, faut-il rappeler qu'en l'espace de quelques années, la définition de critères et d'indicateurs

qualitative ou descriptive, qui, mesurée et surveillée périodiquement, montre la direction du changement ».

${ }^{6}$ Chaque session de cette conférence s'accompagne de la production d'un certain nombre de déclarations et de résolutions générales, que les États signataires s'engagent à appliquer de manière volontaire. Les documents sont consultables sur le site http://www.mcpfe.org 


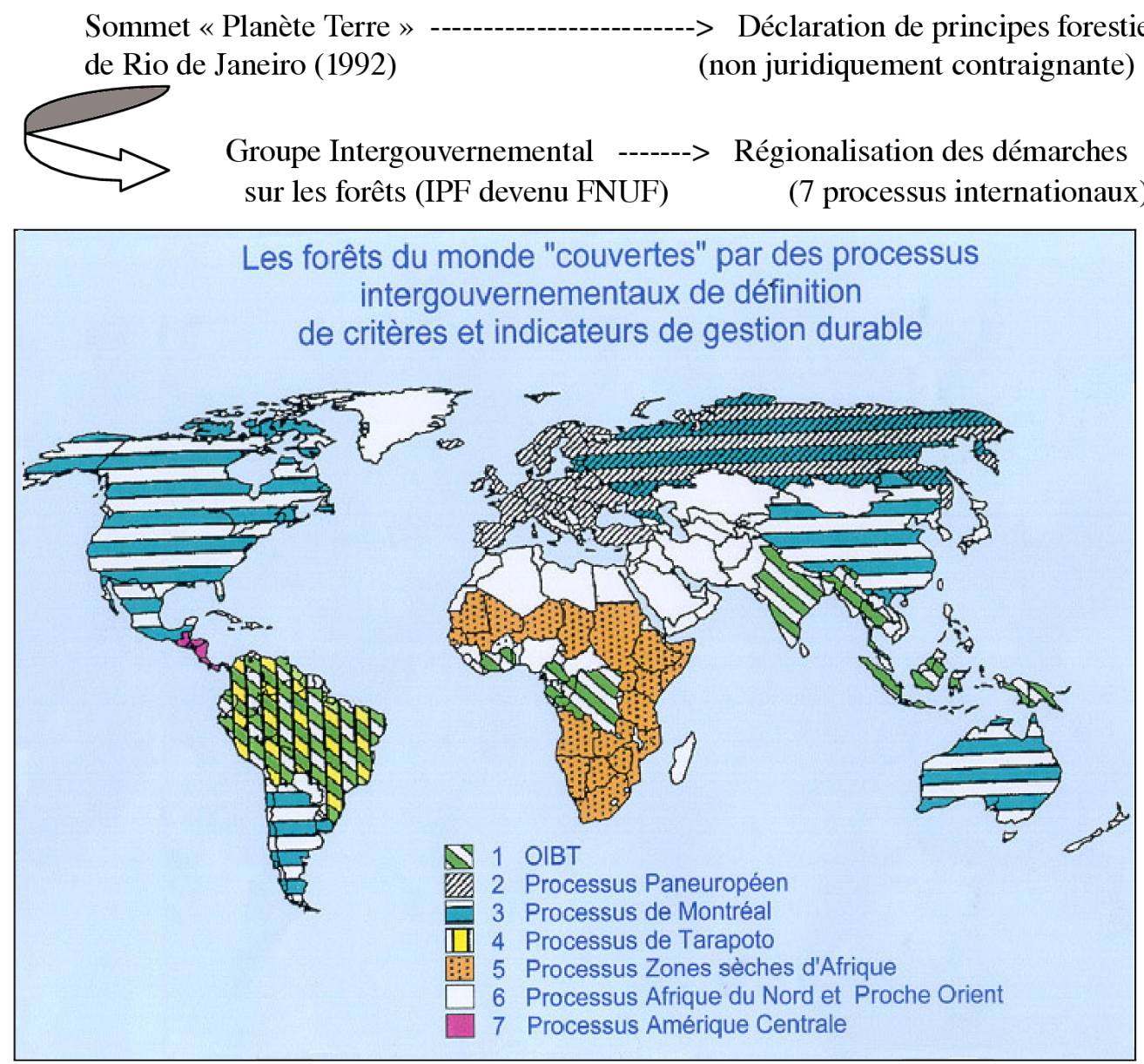

\section{Processus paneuropéen (40 pays membres)}

- Définition révisée de la gestion durable

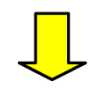

- Jeu global de C \& I

( 6 critères/35 indicateurs)

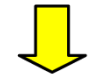

- Recommandations à l'unité de gestion forestière

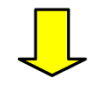

- Programmes nationaux forestiers (approche par écosystèmes)

Fig. 1. La logique de la définition toujours plus précise de la durabilité retenue par les processus intergouvernementaux.

de durabilité, qu'ils soient d'ordre général ou établis pour un secteur spécifique, s'est tant et si bien généralisée que chacun la considère désormais comme parfaitement légitime, naturelle et, pour tout dire, seule possible.

En fait, la démarche par principes, critères et indicateurs de ces processus intergouvernementaux aboutit à la production d'une sorte de vaste tableau de bord. Classés par grands domaines (les critères), les indicateurs (que pour la plupart, on se serait contenté, naguère, d'appeler statistiques) en constituent les multiples paramètres ou voyants informatifs. Ce tableau de bord renseigne d'une part sur l'état général des forêts d'un pays et, d'autre part, sur la nature et la densité des dispositifs d'actions publiques en faveur des forêts. Il permet à la rigueur de suivre un certain nombre d'évolutions d'une année ou d'une période à l'autre, et d'effectuer des comparaisons entre politiques forestières des différents pays. Que les experts des administrations forestières des différents pays membres aboutissent à un tel résultat se situe donc bien dans l'ordre des choses. Et tout irait pour le mieux si cette finalité première des processus intergouvernementaux ne disparaissait sous la prétention d'avoir construit un référentiel établissant ce qu'est la durabilité forestière.

\section{Une quasi-norme environnementale}

Dès lors, la logique intellectuelle de la démarche suivie se révèle beaucoup moins neutre qu'il n'y paraît. À chaque étape, des modifications sont opérées, des options sont prises qui aboutissent in fine à une certaine dénaturation du questionnement initial. On pourrait presque comparer le processus à l'œuvre à celui que matérialisent ces séries de dessins par lesquelles, en un nombre réduit d'étapes, un voilier se transforme en coquillage, une baleine en sirène, une ville en prairie. D'une étape à l'autre, le changement est à peine perceptible ; subtiles, les inflexions ne portent jamais que sur quelques traits ; en fin de course cependant, la métamorphose est bel et bien accomplie (Brédif et Arnould, 2004).

Reprenons. Initialement posée de manière globale à Rio, la question du développement durable se retrouve bientôt segmentée en " grands domaines thématiques » jugés importants : agriculture, tourisme, eau, climat, énergie... Chacun réunit son cénacle de spécialistes : ainsi apparaît la notion de durabilité forestière, son forum spécifique et ses experts attitrés. 
Le champ d'investigation demeurant considérable, un second découpage intervient, à première vue toujours anodin. Cette fois, il opère sur des bases spatiales : la régionalisation de la durabilité forestière aboutit à la création des sept processus gouvernementaux.

Troisième étape et non des moindres, parfaitement incarnée par le processus paneuropéen, le terme de «gestion » remplace celui de «développement $:$ le questionnement sur la durabilité forestière et les conditions de sa réalisation se trouve réécrit en termes de «gestion durable des forêts ». Le processus paneuropéen se dote à ce sujet d'une définition nouvelle (Encadré 1). À cette occasion, un autre changement de vocable intervient, aussi majeur qu'inaperçu : la notion de «fonction » est substituée à celle de «besoins » («besoins à satisfaire») qui occupe une place centrale dans la définition inaugurale du développement durable et avait été reprise telle quelle dans la déclaration de principes forestiers de Rio. Là encore, ces inflexions sont justifiées par la volonté de définir selon des bases tangibles la notion de durabilité forestière.

Une fois réalisées, ces modifications, bénignes en apparence, conjuguent leurs effets pour permettre un formidable tour de passe-passe : la gestion durable des forêts va pouvoir être assimilée à une "gestion multifonctionnelle des forêts ", elle-même identifiée à une manière de «bonne gestion forestière » revisitée au goût du jour - c'est-à-dire une gestion des forêts en bon père de famille, enrichie des préoccupations écologiques de notre temps (sol, climat, biodiversité...) et accessoirement de données sociales (accueil du public). L'enjeu large et ouvert, formulé en termes de satisfaction conjointe de besoins variés relatifs aux forêts, est ramené, pour l'essentiel, à la conservation de l'intégrité des écosystèmes forestiers, afin de leur permettre de continuer à assumer leurs diverses fonctions.

Le passage aux critères et indicateurs aggrave cette inclination, dès lors que, dans un souci d'objectivité forte et de mesure scientifique, il focalise l'attention sur «l'objet forestier lui-même » et les conditions de son intégrité propre, au détriment des acteurs et des besoins que ceux-ci pourraient avoir. En d'autres termes, la durabilité forestière se trouve réduite à la qualité intrinsèque des forêts (Fig. 2).

Au demeurant, ces indicateurs sont bien mal nommés. Ils n'indiquent certainement pas la réalité ou non d'une gestion durable; il serait plus juste d'y voir de simples descripteurs de l'état des forêts et de certaines fonctions ou rôles remplis par les forêts. Bien entendu, aucun $d^{\prime}$ entre eux ne permet de savoir s'il existe ou non une adéquation entre les besoins des acteurs et les situations forestières observées. Quant aux indicateurs qualitatifs de ce processus, ils ne sont pour le moment que très peu approfondis et renvoient avant tout à l'existence de dispositifs institutionnels susceptibles d'assurer la gestion de telle ou telle fonction.
Encadré 1. Définition de la gestion durable par le processus paneuropéen

La première résolution de la Conférence ministérielle pour la protection des forêts d'Europe tenue à Helsinki en 1993 énonce une série de principes généraux pour la gestion durable des forêts, parmi lesquels : « la priorité donnée à éviter les dégradations irréversibles des sols et de la diversité biologique, l'importance reconnue à la forêt privée et au soutien des actions des propriétaires-sylviculteurs, la reconnaissance du rôle des aménagements... ». Les négociateurs français, Christian Barthod et Georges Touzet (1994), se félicitent surtout du fait que cette résolution donne pour la première fois une définition de la gestion durable, entendue comme « la gérance et l'utilisation des forêts et des terrains boisés, d'une manière et à une intensité telles qu'elles maintiennent leur diversité biologique, leur productivité, leur capacité de régénération, leur vitalité et leur capacité à satisfaire, actuellement et pour le futur, les fonctions écologiques, économiques et sociales pertinentes, aux niveau local, national et mondial, et qu'elles ne causent pas de préjudices à d'autres écosystèmes ».

Poursuivie jusqu'à formuler des recommandations pour la planification de la gestion forestière et pour les pratiques de gestion à l'échelle de l'unité de gestion forestière, cette approche revient à définir in abstracto une sorte de forêt idéale, valable en tout temps, à toutes échelles et en tout lieu.

Ainsi, et sans que cela ne corresponde probablement à une quelconque stratégie ou intention délibérée, le questionnement de la durabilité s'est mué en quête de référentiel technico-administratif de bonne gestion forestière. Un référentiel auquel les conférences ministérielles confèrent une pleine légitimité et auquel la reprise à l'identique ou presque par les autres processus gouvernementaux confère un statut universel.

Toutefois, des impasses logiques se font bientôt jour ; à cet égard, les recommandations de Lisbonne s'avèrent édifiantes. Poussée à l'échelle de l'unité de gestion, la logique des principes, critères et indicateurs impose à tout propriétaire ou gestionnaire forestier : (i) d'accueillir du public; (ii) de produire du bois et de contribuer à l'économie de la filière et à l'emploi ; (iii) de favoriser le maintien de la biodiversité et de lutter contre le changement climatique. Or, cette standardisation de la gestion et des objectifs ne correspond pas aux aspirations des acteurs. Par exemple, les associations de conservation de nature revendiquent pour certaines forêts de laisser les choses évoluer par elles-mêmes, en évitant autant que faire se peut toute forme de perturbation. Quant aux propriétaires forestiers, ils ont le sentiment que leur liberté est brimée dans des carcans et des réponses univoques, là où la diversité des comportements et des postures constitue de facto une incomparable garantie de diversité. 


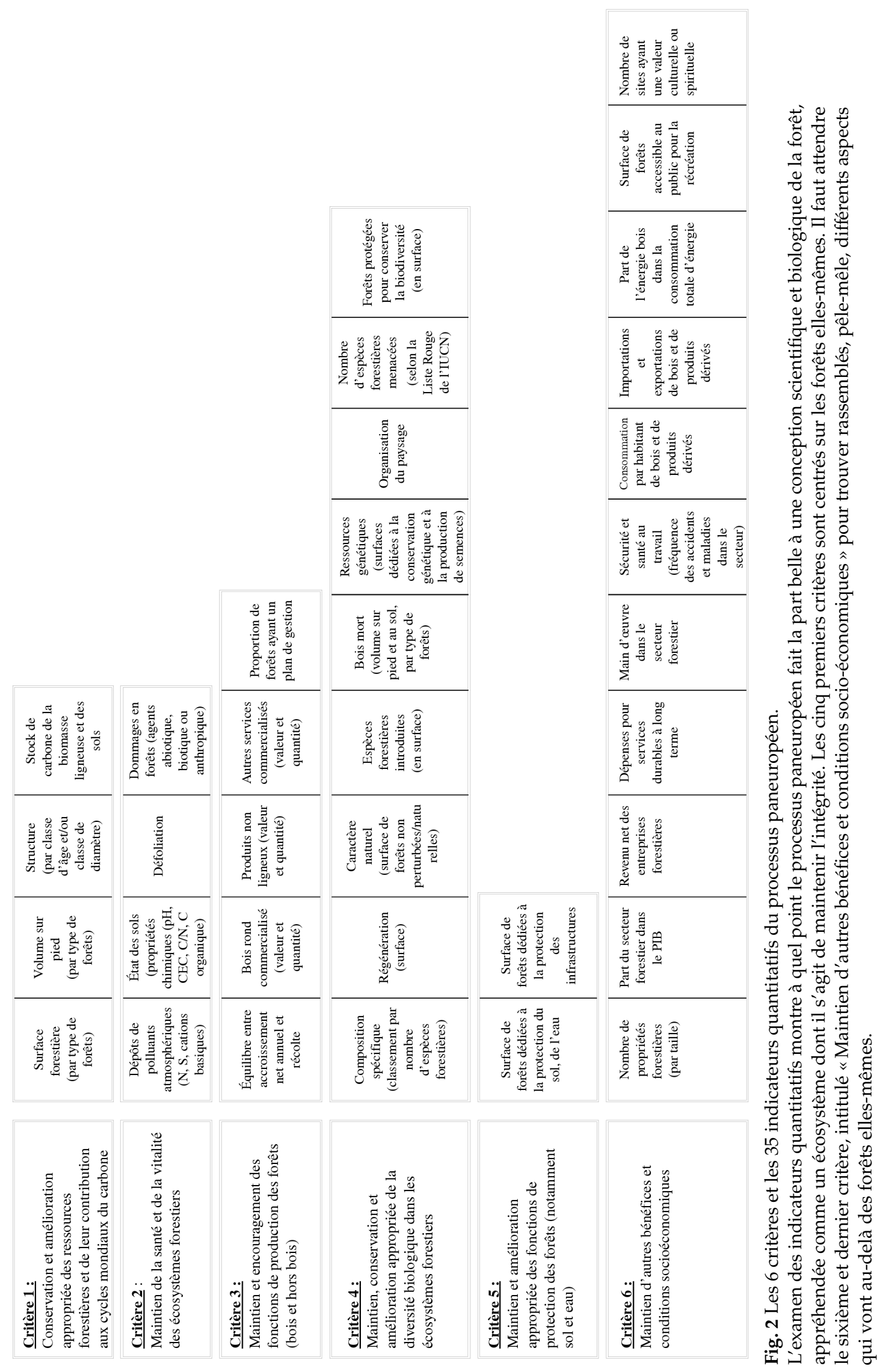


Encadré 2. Recommandations paneuropéennes pour une gestion forestière durable au niveau opérationnel. Extrait du critère 3 : «Maintien et encouragement des fonctions de production des forêts (bois et hors bois) »

3.2. Recommandations pour les pratiques de gestion forestière

a. La qualité des pratiques de gestion forestière devrait être assurée dans le but de préserver et d'améliorer les ressources forestières et d'encourager à long terme une production diversifiée de biens et services.

b. Les opérations de régénération, d'entretien et d'exploitation devraient être réalisées en temps opportun et de manière à ne pas réduire la capacité productive du site, par exemple, en évitant de causer des dégâts dans les peuplements et arbres restants, de même qu'aux sols des forêts, grâce à l'emploi de moyens adéquats.

c. Les niveaux d'exploitation des produits, tant ligneux que non ligneux, ne devraient pas dépasser un rythme tel qu'il puisse être maintenu à long terme et il conviendrait de faire un usage optimal des produits forestiers exploités en tenant dûment compte du prélèvement de nutriments.

d. Il conviendrait de planifier, créer et entretenir une infrastructure adéquate, telle que routes, pistes de débardage ou ponts, afin d'assurer un écoulement efficace des biens et services tout en minimisant les impacts négatifs sur l'environnement.

De surcroit, en y regardant de plus près, les recommandations de Lisbonne s'avèrent surtout précises pour ce qui relève de l'état écologique de la forêt, de la diversité des essences et de l'accueil du public. Le domaine économique, trop dépendant de paramètres exogènes à la forêt, est difficilement accessible à des préconisations de cet ordre. De sorte que la gestion durable, in fine, s'apparente de plus en plus à une norme technique dominée par des préoccupations environnementales. L'examen des recommandations opérationnelles relatives au critère 3 (Encadré 2) - le seul vraiment dédié aux considérations économiques - le montre bien : celles-ci visent avant tout à recommander d'utiliser les propres capacités productives de «l'écosystème forestier », en limitant au maximum les impacts sur l'environnement.

La problématique de la durabilité ${ }^{7} \mathrm{~s}^{\prime}$ est dissoute dans le référentiel technico-administratif susceptible de garantir des forêts intrinsèquement bien gérées.

\footnotetext{
7 Eu égard à la définition inaugurale du développement durable retenue par le Sommet de la Terre, mettant l'accent sur la notion de «besoins à satisfaire ». Il existe certes d'autres acceptions de la durabilité forestière dans la littérature scientifique nous avons nous-mêmes contribué à en dresser une typologie (Brédif et Boudinot, 2001) - mais cela ne change rien à ce que nous cherchons à montrer dans cet article, à savoir la dérive d'un processus intergouvernemental qui prétend pourtant s'inscrire dans le sillage du Sommet de la Terre.
}

\section{Toujours plus de la même chose : la certification forestière}

Les écrits sur la certification forestière sont souvent prisonniers d'une vision manichéenne qui oppose un premier système, celui du Forest Stewardship Council (FSC), soutenu par la plupart des ONG environnementales, à des dispositifs conçus par des professionnels de la forêt et du bois, au premier rang desquels se trouve le PEFC (Pan-European Forest Certification Scheme). Une véritable guerre des labels et des marques s'ensuit, chaque groupe d'influence impliquant des énergies colossales, dans un affrontement en passe d'accéder à une dimension planétaire.

À grands renforts de lobbying, de pressions sur les donneurs d'ordres, d'enrôlements de stars du show business, les partisans du FSC tentent d'imposer cet organisme et sa marque comme références absolues. Dans une déclaration conjointe de 2001, près de cinquante associations de protection de la nature ont clairement pris position en faveur du FSC ${ }^{8}$, estimant qu'il s'agissait du «seul système de certification crédible et indépendant existant à l'heure actuelle, susceptible d'apporter des garanties fiables quant à l'origine "gestion durable des bois » ayant servi à fabriquer tel ou tel article, qu'il s'agisse d'un meuble, d'un crayon ou d'une ramette de papier. Le soutien inconditionnel du WWF, de Greenpeace et de la plupart des ONG de rang international constitue un argument de poids, avec lequel les groupes industriels et les grands distributeurs sont obligés de compter, tandis que le « risque de réputation » devient une donnée cardinale de la stratégie des entreprises.

En face se trouvent les représentants des propriétaires forestiers et de leurs organisations, désireux de rester maîtres en leur domaine et refusant le monopole d'un système étroitement lié aux ONG. Dix millions de propriétaires en Europe constituent également une force incontournable, d'autant que le PEFC, sollicité par des acteurs forestiers d'autres régions du monde, tend à devenir un système générique susceptible d'accueillir les démarches de certification forestière issues de professionnels du monde entier. Rebaptisé dernièrement «Programme for the Endorsement of Forest Certification Schemes », le PEFC est en effet transformé en mécanisme international pour la reconnaissance mutuelle de démarches volontaires de certification ${ }^{9}$.

\footnotetext{
8 On notera cependant que France Nature Environnement, la Fédération française des associations de défense de l'environnement, ne s'est pas jointe au mouvement général. Elle est d'ailleurs partenaire de la démarche PEFC France.

${ }^{9}$ La plupart des démarches de certification issues des professionnels de la forêt et du bois ont désormais rejoint le PEFC. Il s'agit en particulier de : CSA (Canadian Standard Association), SFI (Sustainable Forestry Initiative in the Unites States), CERFLOR system au Brésil, le CERTFLOR au Chili, le MTCC en
} 
La situation de rivalité aiguë qui résulte de l'opposition entre le FSC et le PEFC accapare l'attention et empêche souvent toute prise de recul. Tantôt, les analystes comptabilisent les points en comparant scrupuleusement les avantages, les réussites et les déficiences de chaque système, tantôt ils espèrent pouvoir passer outre leurs antagonismes au nom d'un idéal d'harmonisation. Dans la fureur de l'affrontement, peu de personnes trouvent le temps de s'interroger sur la légitimité des cahiers des charges, à savoir la référence «gestion durable» sur laquelle s'appuient ces différents systèmes de certification forestière. Ainsi, la matrice de comparaison établie par le Confédération européenne des industries papetières (CEPI, 2000) passe-t-elle au crible la plupart des aspects relatifs à la structure des dispositifs de certification/labellisation et aux procédures qu'ils impliquent; en revanche, elle esquive largement la question de la référence "gestion durable », élément pourtant névralgique de l'ensemble de la démarche de certification puisqu'il en constitue à proprement parler le socle fondamental, sur la base duquel statue le certificateur. C'est donc sur ce point précis qu'il convient de s'arrêter. Auparavant, un retour sur les conditions d'émergence de l'idée de certification forestière s'impose.

\section{Avènement d'un nouveau type d'acteur global}

Les organisations non gouvernementales comptent parmi les grands gagnants de la mondialisation. Présentes sur tous les fronts, nul ne s'étonne plus de leurs succès. Elles incarnent pour beaucoup le rôle de vigies, seules à même d'exercer un véritable contre-pouvoir face aux intérêts des puissants (Pech et Padis, 2004). Reconverti dans l'humanitaire, un éminent observateur invité dernièrement à la tribune des Nations unies ${ }^{10}$ a célébré l'avènement de ce nouvel acteur global dédié au «bien commun de l'humanité ». Selon l'auteur de cette apologie, ces entités nouvelles, qu'il propose de renommer Institutions de solidarité (IDS), «pèsent plus lourd sur le destin de l'humanité que bien des nations ayant leur siège dans cette salle » [...]; «elles constituent un formidable outil de transformation économique, politique, sociale, culturelle, de la planète [...]; elles sont en train de créer une nouvelle dynamique transfrontière, nomade, qui sera un jour plus puissante que celle du marché et de la politique». Les ONG seraient donc l'avenir de l'humanité, car elles seules s'occupent des causes perdues, quand le marché n'a jamais prétendu s'y intéresser et les États ne savent pas comment les aborder.

Malaisie et l'Australian forest certification and the Pan-African Forest Certification Scheme (PAFC) basé au Gabon.

${ }^{10}$ Il s'agit de Jacques Attali, président en titre d'un organisme de microcrédit, PlaNet Finance. Les citations proviennent d'un article paru dans Le Monde, le 11 septembre 2006 : « Les ONG, rempart contre l'apocalypse », reprise du discours de clôture du sommet mondial des ONG, prononcé par Jacques Attali à la tribune des Nations unies le 10 septembre 2004.

\section{Un changement majeur de paradigme : l'écologie par le marché}

L'idée même de certification forestière témoigne d'une rupture idéologique considérable au sein des ONG de défense de l'environnement. L'économique et le marchand ne sont en effet plus considérés comme étant la source de la dégradation de l'environnement terrestre, ainsi que le voulait une conception antérieure. Désormais, la réalité du marché n'est pas seulement acceptée, elle est surtout envisagée comme un levier puissant pour parvenir à des fins écologiques. Ayant perdu confiance dans la volonté et la capacité des gouvernements à infléchir le cours des choses, dubitatives devant la possibilité de mobiliser activement et dans la durée les citoyens ordinaires pour de grandes questions environnementales, les ONG placent désormais leurs espoirs dans une nouvelle arme stratégique : le marché lui-même ${ }^{11}$. Cette forme de pragmatisme n'est sans doute pas étrangère à l'aire culturelle, anglosaxonne, à laquelle ressortissent la plupart des ONG de conservation de la nature de rang international. En tout cas, il convient de souligner que ces institutions font preuve d'une véritable capacité de réflexion et d'innovation stratégiques, mue par un réel désir de changement et d'action. Partant du constat que les modes d'intervention classiques plafonnaient dans leurs effets comme dans les moyens financiers dont ils disposaient, certaines ONG de rang international en ont cherché de nouveaux. C'est ainsi qu'elles ont découvert la certification.

Au départ, il s'agissait de trouver un moyen de garantir à tout consommateur qu'il ne contribuait pas, par son acte d'achat de produits à base de bois, à encourager, même à son insu, la dégradation des forêts du monde. En d'autres termes, cela revenait à donner à tout acheteur potentiel une capacité de peser sur l'évolution de la situation des forêts du monde. À cet égard, le mécanisme de la certification forestière paraît, à première vue, aussi simple que probant.

Certifier signifie « attester qu'une chose est vraie». Pour authentifier un propos, une œuvre d'art, une signature, trois composantes sont nécessaires et suffisantes : 1) une référence reconnue comme telle ;2) une procédure transparente et opposable permettant de statuer sur la conformité entre ladite référence et le candidat à l'authentification; 3) enfin, une tierce partie, indépendante et neutre, capable de soumettre en toute impartialité le candidat à l'épreuve de la conformité. Courante et pratiquée depuis des lustres dans de nombreux compartiments de la vie en société, cette procédure n'avait toutefois jamais été appliquée à une matière aussi spéciale que la durabilité forestière (Brédif et Arnould, 2004). Plusieurs

\footnotetext{
11 "Je suis une fille matérialiste dans un monde matérialiste», clamait, dans les années 1990, une célèbre pop star. De même, les ONG pourraient-elle dire que, dans un monde dominé par l'utilitaire et le marchand, elles ne pouvaient pas rester hors du courant dominant.
} 
ONG, le WWF en tête, n'ont pas hésité longtemps à la transposer. Ainsi, le Forest Stewardship Council, qu'elles ont largement contribué à créer au début des années 1990, s'est-il doté d'une sorte de cahier des charges de la gestion durable des forêts - la référence - , constituée de dix principes généraux, réputés universels, complétés de quelque cinquante-six critères. Il restait à trouver des organismes certificateurs se chargeant de vérifier l'adéquation entre les situations forestières réelles - candidates à la certification - et le cahier des charges, tout en précisant les modalités de la procédure en question. Une fois les forêts certifiées, il convenait enfin d'assurer un suivi du bois issu desdites forêts dans l'ensemble du processus industriel de transformation, afin de garantir au consommateur final l'innocuité du produit acheté - c'est là qu'intervient la question technique de la chaîne de contrôle et de la labellisation des produits.

Le grand tour de force du dispositif réside dans le fait qu'il repose sur une démarche volontaire et permet ainsi de s'affranchir de toute interférence étatique. Il en résulte un mécanisme privé, tiré par le marché (Cashore et Bernstein, 2005), dans un secteur forestier où jusqu'alors la régulation publique s'avérait prépondérante.

\section{L'universalité, encore une fois mise en avant}

Aux dires de ses promoteurs, le Forest Stewardship Council est un «système mondial, applicable à toutes les régions et à toutes sortes de régimes fonciers ». Une qualité à laquelle ne sauraient prétendre les autres systèmes de certification forestière. En somme, il s'agirait d'un système résolument universel : « qui vaut pour tout et pour tous ». Quant aux autres systèmes, notamment le PEFC, ils présenteraient l'inconvénient majeur de n'avoir été pensés que pour des situations locales ou régionales. Cette prétention à l'universalité du système FSC apparaît dans les documents fondateurs de l'organisme et constitue toujours un axe de communication majeur (FERN, 2001). Sur quoi s'appuie-t-elle? En premier lieu, elle est censée émaner des dix grands principes du FSC. Ceux-ci seraient libellés de telle manière qu'ils demeureraient pertinents en tous lieux, en tous temps et à toutes les échelles. De surcroît, les modalités ayant présidé à leur établissement renforceraient leur caractère universel. À la différence des processus intergouvernementaux qui réunissent principalement des délégués des différents pays, les dix principes du FSC résultent d'une négociation entre les trois collèges de forces vives. Le caractère privé de la démarche - par opposition au caractère officiel des processus gouvernementaux - , la pluralité des intérêts en présence et surtout le caractère consensuel des dix principes du FSC sont présentés comme autant de gages d'universalité. Sans qu'il soit question de verser dans le camp des détracteurs qui rejettent toute légitimité au FSC, faute de s'appuyer sur un processus gouvernemental, il convient cependant de s'interroger sur la validité des arguments avancés. Car il ne suffit pas de dire que les autres systèmes ont été élaborés pour des aires d'application restreintes pour qu'il en découle, par contraste, une authentique universalité des dix principes du FSC. En fait, trois raisons majeures peuvent être invoquées à l'encontre de cette prétention à l'universalité.

\section{Encore plus loin dans le prescriptif et dans la qualité intrinsèque}

Les critiques formulées à l'encontre des jeux de critères et indicateurs des processus gouvernementaux doivent être reconduites et même amplifiées dans le cas de la certification. Certifier suppose en effet de pouvoir attester la concordance entre une situation concrète et une référence considérée comme « modèle à suivre ». Par construction, la procédure de certification requiert donc l'élaboration d'un référentiel suffisamment précis pour que l'auditeur extérieur - le certificateur - puisse établir sans coup férir s'il y a, ou non, conformité. Il ne doit pas y avoir de place pour des marges d'appréciation. Le référentiel de certification forestière ne peut donc pas se contenter de recommandations plus ou moins générales. Pour pouvoir se prononcer de manière certaine, le certificateur a besoin d'un référentiel aussi stable et prescriptif que possible. Par rapport aux processus gouvernementaux, la différence ne se situe donc pas tant dans l'approche - finalement très comparable, puisque, là aussi, on cherche à définir a priori ce qu'est la gestion durable en recourant à des principes et des critères - que dans le degré de vérification auquel on soumet les situations forestières. On pourrait presque dire que la certification forestière va jusqu'au bout de la logique des processus intergouvernementaux. Elle permet de réaliser ce que les États n'ont osé faire. Cette remarque s'applique à tous les systèmes de certification forestière. Il est un point cependant sur lequel le système du Forest Stewardship Council enfonce le clou. En insistant sur l'importance des niveaux de performance à atteindre à l'échelle de l'unité de gestion forestière à certifier - nombre d'arbres morts à l'hectare, taille des coupes rases, proportion des espaces forestiers dédiés à un objectif de protection stricte... - , il parachève sans complexe la «logique substantielle » de la durabilité. Dès lors, la problématique de la gestion durable des forêts se dissout dans une norme de procédure, surmontée d'une norme de performance où prédominent les critères sociaux et environnementaux. Les derniers documents publiés par le WWF le reconnaissent explicitement puisqu'ils présentent désormais la marque FSC comme une garantie de «gestion écologiquement et socialement responsable » (WWF, 2005). 


\section{Les grandes unités forestières avantagées}

Au demeurant, que penser des principes du FSC ? Sont-ils aussi neutres et universels qu'on le prétend? Un examen attentif suggère, au contraire, qu'ils promeuvent de fait certaines approches de la gestion durable plutôt que d'autres. Pour s'en convaincre, il suffit de consulter les chiffres. Qui sont les grands bénéficiaires des systèmes de certification, de la certification FSC en particulier? Les plantations et les multinationales de la forêt et du bois ! Et cela est normal, car en distinguant les plantations des autres forêts - le fameux principe $10^{12}$-, le système FSC donne l'avantage à un modèle forestier monofonctionnel... au détriment des autres modèles forestiers, notamment les modèles dits multifonctionnels qui prévalent en Europe de l'Ouest. De 2000 à 2005 par exemple, les plantations industrielles du Brésil ont représenté les deux tiers des surfaces certifiées par le FSC. Plus généralement, de nombreux travaux (Buttoud et Karsenty, 2001; Atyi et Simula, 2002; Ozanne et Vlosky, 2003; FAO, 2007) indiquent que les forêts certifiées - tous systèmes confondus - se trouvent à plus de $80 \%$ dans l'hémisphère Nord (Europe et États-Unis), avec une part prépondérante pour les plantations. En fait, la mise en œuvre des critères de certification s'avère plus rentable et plus facile dans le cas de grandes unités forestières, là où opèrent des groupes industriels ; il s'avère par exemple plus aisé de distraire quelques centaines ou milliers d'hectares mis en réserve intégrale dans le cas de massifs forestiers de plusieurs dizaines ou centaines de milliers d'hectares gérés par un seul opérateur - afin de satisfaire à certains niveaux de performance, le reste étant dévolu à un objectif dominant de production - que dans le cas d'une propriété forestière fragmentée.

\section{Les besoins des acteurs et la réalité des situations sont oubliés}

Les démarches de certification révèlent une autre aporie de l'approche consistant à définir a priori et de manière universelle un contenu à la durabilité forestière. Le comprendre suppose de se placer cette fois sur le plan de l'efficacité de l'action. Dans ces différentes approches, qu'il s'agisse des processus intergouvernementaux ou bien des dispositifs de certification, la mise aux normes « gestion durable » revient aux seuls propriétaires et gestionnaires forestiers, sans que cela ne leur rapporte bien souvent quoi que ce soit. On fait comme si ces derniers disposaient d'emblée des moyens techniques, financiers

\footnotetext{
12 Nous ne reproduisons pas ici les dix principes et les 56 critères du FSC, qui peuvent être aisément trouvés sur la Toile et n'apportent pas foncièrement de nouveauté par rapport aux critères et aux indicateurs présentés dans le cas du processus paneuropéen ; la différence tient au fait que critères et niveaux de performance dans le cas du FSC s'avèrent plus exigeants.
}

et humains de se mettre en conformité avec le nouveau référentiel. Il en résulte une curieuse asymétrie : chaque partie concernée par les forêts se voit accorder le droit de définir ce qui est souhaitable, mais ensuite l'effort s'avère largement unilatéral. En somme, propriétaires et gestionnaires forestiers se retrouvent à devoir faire ce que les autres n'ont qu'à dire. Et cela, au nom du bien commun!

En outre, en évacuant le questionnement sur la possibilité de satisfaire conjointement les différents besoins relatifs aux forêts ${ }^{13}$, ces démarches perdent de vue la réalité des situations, des territoires et des problèmes. Là où précisément se posent des problèmes de compatibilité entre usages et objectifs, là où se joue la délicate question des articulations entre échelles, elles se contentent de répondre par un modèle standard, comme si la durabilité fonctionnait à la manière d'un simple puzzle.

\section{Conclusion}

Dans son ouvrage très stimulant, Jared Diamond ne se contente pas de dégager les grands facteurs explicatifs du maintien ou de la disparition des sociétés. L'approche comparative qu'il adopte lui permet in fine d'identifier les deux conditions, essentielles à ses yeux, pour que les sociétés perdurent ${ }^{14}$. Ces deux conditions constituent en fait une tentative de traduction opérationnelle du cinquième facteur « la capacité des sociétés à faire face à leurs problèmes, en y apportant des réponses adaptées ».

Malheureusement, la question demeure entière de savoir ce que sont des réponses adaptées. Dans le cas de la durabilité forestière, la volonté des États, des ONGE, d'autres acteurs aussi, d'améliorer le cours des choses semble hors de cause. Pour autant, les développements précédents le montrent, les référentiels auxquels ils aboutissent ont dérivé par rapport au questionnement initial du développement durable : en l'état, ils ne constituent certainement pas une réponse et encore moins une solution universelles.

Ces quelques observations suggèrent que l'ensemble des conditions nécessaires à la mise en œuvre d'une véritable durabilité forestière n'a toujours pas été reconnu. Pour parler comme Grégory Bateson (1979), une réflexion reste à conduire sur les conditions de la durabilité, dans laquelle une plus grande importance serait accordée à des démarches réflexives et ascendantes (Voss et al., 2006).

\footnotetext{
${ }^{13}$ Dont nous avons dit plus haut qu'il correspondait à la problématique cardinale portée par la définition inaugurale du développement durable.

14 Anticipation à long terme et capacité de remettre en cause les valeurs fondamentales sur lesquelles reposent les sociétés correspondent aux deux conditions identifiées par Diamond en page 522 de son ouvrage.
} 


\section{Références}

Atyi, R., Simula, M., 2002. Forest Certification: Pending Challenges for Tropical Timber, IITO Technical Series, 19.

Austin, J. L., 1970. Quand dire, c'est faire, Paris, Seuil.

Barthod, C, Touzet, G., 1994. De Strasbourg à Helsinki. Les deux premières conférences ministérielles pour la protection des forêts en Europe, Revue Forestière Française, 46, 4, 319-334.

Bateson, G., 1979. La nature et la pensée, Paris, Seuil.

Brédif, H., Boudinot, P., 2001. Quelles forêts pour demain? Éléments de stratégie pour une approche rénovée du développement durable, Paris, L'Harmattan.

Brédif, H., Arnould, P., 2004. Évaluer n'est pas gérer. Considérations pour rompre le pouvoir des critères et des indicateurs, Revue Forestière Française, 56, 5, 485-502.

Buttoud, G., Karsenty, A. (2001). L'écocertification de la gestion des forêts tropicales, Revue forestière française, 53, 6, 691-706.

Cashore, B., Bernstein, S., 2005. Legitimacy and the privatization of environmental governance: How Non-State MarketDriven (NSMD) governance systems gain rule-making authority, Governance: an international journal of policy, administration and institutions, 15, 4, 503-529, Blackwell Publishing.

CEPI (Confederation of European Paper Industries), 2000. Comparative Matrix of Forest Certification Schemes. Bruxelles.

Diamond, J., 2005. Collapse. How societies choose to fail or succeed. New York, Penguin Books.

Reçu le 20 avril 2007. Accepté le 27 juin 2008.
FAO (Food and Agriculture Organization), 2006. Forest Products Annual Market Review 2005-2006, Rome.

FAO, 2007. Situation des forêts du monde 2007, Rome.

FERN (the Forests and European Union Resource Network), 2001. Behind the logo, An environnemental and social assessement of forest certification schemes. Rapport établi à partir d'études de cas de WWW France, Taiga Consulting, Taiga Rescue Network, Robin Wood, NRDC, Fern, Finnish Nature League, Greenpeace International.

Guéneau, S., 2007. Certification as a new private global forest governance system: The regulatory potential of the Forest Stewardship Council. Communication orale effectuée le 8 février 2007 au Basel Institute on governance.

Humphreys, D., 2006. Logjam. Deforestation and the crisis of global governance. Londres, Earthscan.

Ozanne, L.K., Vlosky, R.P., 2003. Certification from the US consumer perspective: A comparison from 1995 and 2000, Forest products journal, 53, 3, 13-21.

Pech, T., Padis M.-O., 2004. Les multinationales du cour : les ONG, la politique et le marché, Paris, Seuil.

Voss, J.-P., Bauknecht, D., Kemp, R., 2006. Reflexive governance for sustainable development, Cheltenham, Edward Elgar Publishing.

WWF (World Wide Fund For Nature), 2005. Rapport Forests for Life 2005, Gand, WWF international. 